\title{
Dislocation of the Elbow: A Retrospective Multicentre Study of 86 Patients
}

\author{
Jeroen de Haan ${ }^{1}$, Niels W.L. Schep ${ }^{2}$, Imme Zengerink ${ }^{3}$, Jesse van Buijtenen ${ }^{1}$, Wim E. Tuinebreijer ${ }^{4}$ \\ and Dennis den Hartog ${ }^{*}, 2$
}

\author{
${ }^{I}$ Department of Surgery and Traumatology, Westfriesgasthuis, Maelsonstraat 3, 1624 NP Hoorn, The Netherlands \\ ${ }^{2}$ Department of Surgery - Traumatology, Erasmus MC, University Medical Center Rotterdam, P.O. Box 2040, \\ 's Gravendijkwal 230, 3015 CE Rotterdam, The Netherlands \\ ${ }^{3}$ Department of Surgery, Reinier de Graaf Gasthuis, Reinier de Graafweg 3-11, 2625 AD Delft, The Netherlands \\ ${ }^{4}$ Relweg 59, 1949 EC Wijk aan Zee, The Netherlands
}

\begin{abstract}
The objective of this retrospective multicentre cohort study was to prospectively assess the long-term functional outcomes of simple and complex elbow dislocations.

We analysed the hospital and outpatient records of 86 patients between 01.03.1999 and 25.02.2009 with an elbow dislocation. After a mean follow-up of 3.3 years, all patients were re-examined at the outpatient clinic for measurement of different outcomes.

The mean range of motion was ROM 135.5 . The Mayo elbow performance index (MEPI) scored an average of 91.9 ( $87.5 \%$ of the patients were rated excellent or good). The average Quick disabilities of the arm, shoulder and hand (QuickDASH) score was 9.7, the sports/music score 11.5 and work score 6.1. The Oxford function score was 75.7, Oxford pain score 75.2 and Oxford social-psychological score 73.9.
\end{abstract}

Elbow dislocation is a mild disease and generally, the outcome is excellent. Functional results might improve with early active movements.

Keywords: Elbow, elbow joint, injury, dislocations.

\section{INTRODUCTION}

The elbow joint is the second most commonly dislocated joint in adults. The annual incidence of simple and complex elbow dislocations in children and adults is 6.1 per 100,000 [1]. Elbow dislocations can be classified as simple or complex [2]. The simple dislocation is characterized by the absence of fractures, while the complex dislocation is associated with fractures. The terrible triad is an example of a complex posterior dislocation with intra-articular fractures of the radial head and coronoid process. The annual incidence of complex elbow dislocations in children and adults is 1.6 per 100,000 , or $26 \%$ percent of all elbow dislocations [1]. After reposition of the simple dislocation, treatment options include immobilisation in a static as well as a functional plaster for different periods, surgical treatment of the ruptured medial and lateral collateral ligaments or so-called functional treatment, which is characterised by early active movements within the limits of pain with or without the use of a sling or hinged brace. The primary objective of this retrospective multicentre observational study was to assess the functional outcomes of simple and complex elbow dislocations. The long-term

*Address correspondence to this author at the Department of Surgery Traumatology, Erasmus MC, University Medical Center Rotterdam, Office H-960, 's-Gravendijkwal 230, 3015 CE Rotterdam, The Netherlands;

Tel: +31 10 7032395; Fax: +31 10 7032396;

E-mail: d.denhartog@erasmusmc.nl outcomes were collected prospectively and assessed with different questionnaires for the upper extremity.

\section{PATIENTS AND METHODOLOGY}

We conducted a retrospective multicentre study of elbow dislocations. All charts of elbow dislocations treated inhospital and at the outpatient departments at three teaching hospitals in the Netherlands from 01.03.1999 to 25.02.200 were collected. Eighty-six cases were identified. Forty-two patients returned for a long-term follow-up examination at the outpatient clinic and an additional 17 patients only filled in the questionnaires. At follow-up range of motion of the elbow was recorded (extension, flexion, pronation, and supination). Each elbow was evaluated using the Mayo elbow performance index (MEPI), a visual analogue scale for pain (VAS), the Quick-DASH (Disabilities of the arm, shoulder, and hand) and the Oxford elbow score.

The MEPI includes five points of interest: Pain (with a maximum score of 45 points), ulnohumeral motion (20 points), stability (10 points) and the ability to perform five functional tasks ( 25 points). If the total score is included between 90 and 100 points, the result was excellent; with a score between 75 and 89 points: Good; between 60 and 74 points: Fair and poor when under 60 [3].

Pain level was determined using a 10-point Visual Analogue Scale (VAS), in which 0 implies no pain and 10 implies the worst possible pain. 
The Quick-Disabilities of the Arm, Shoulder and Hand (DASH) Outcome Measure is a validated 30-item, self-report questionnaire designed to describe the disability experienced by people with upper-limb disorders $[4,5]$. The DASH outcome measure includes three components: The disability/symptom section (11 items, scored 1-5) and two optional modules: One for work and one for sport/music (each optional module has four items, scored 1-5). The DASH disability/symptom score is a summation of the responses to 11 questions on a scale of one to five, with a total score ranging from zero (no disability) to 100 (severe disability). The questions evaluate the degree of difficulty in performing a variety of physical activities with arm, shoulder, or hand problems (six items). They also investigate the severity of pain and tingling (two items), as well as the effect of the upper limb problem on social activities, work and sleep (three items). A higher score indicates greater disability.

The Oxford elbow score is a 12-item questionnaire [6]. It comprises three one-dimensional domains: "elbow function", "pain" and "social-psychological", with each domain comprising four items with good measurement properties [7]. Every question can be answered using a five-point Likert scale, corresponding to a score between zero and four. Every dimension's score is converted to a score of zero (worst) to 100 (best). A Dutch version of a UK validated questionnaire was obtained using the technique of translation and backtranslation [8].

\section{Statistical Analysis}

The statistical analysis was performed with PASW Statistics 17.0 on a personal computer. All proportional data are presented as percentages and all continuous data are given as means with standard deviations (SDs) when normally distributed, and otherwise as medians with the interquartile range (IQR).

The Student independent t-test was used to compare the range of motion for the patients with and without fractures. The Pearson correlations were calculated between the duration of plaster immobilisation, ranges of motion, the MEPI, Quick DASH, VAS and Oxford elbow scores.

\section{RESULTS}

Between 01.03.1999 and 25.02.2009, 86 elbow dislocations were found in 86 patients: 53 were women and 33 men (Table 1). The mean age of these patients was 44 years $(\mathrm{SD}=16)$, with a range of 18 to 84 years. The mechanism of injury was a fall in $89 \%$ of cases $(n=74)$, direct trauma in $8 \%(\mathrm{n}=7)$ and not recorded in five patients. The reason for the trauma was related to sports in $29 \%$ of cases $(\mathrm{n}=18)$, traffic in $20 \%(\mathrm{n}=12)$, an accident at home in $26 \%(\mathrm{n}=16)$, work in $5 \%(\mathrm{n}=3)$, violence in $5 \%(\mathrm{n}=3)$ and leisure in $15 \%(\mathrm{n}=9)$, and in 25 patients it was not recorded.

The type of dislocation was anterior in $1 \%$ of patients $(n=1)$, posterior in $44 \%(n=30)$, posteromedial in $9 \%(n=6)$, posterolateral in $46 \%(n=31)$ and in 18 cases the type of dislocation was missing. Four patients $(5 \%)$ sustained polytrauma. In $69 \%(n=59)$ of the cases the dislocations occurred on the left side. In $89 \%(n=41)$ of the cases in which dominance was recorded $(n=46)$, it was on the right.
Table 1. An Overview of the Patient Population $(n=86)$

\begin{tabular}{|c|c|}
\hline \multicolumn{2}{|l|}{ Sex } \\
\hline Female & $53(61.6 \%)$ \\
\hline Male & $33(38.4 \%)$ \\
\hline Mean age in years & $43.8(\mathrm{SD}=16.2)$ \\
\hline \multicolumn{2}{|l|}{ Cause of injury ( $\mathrm{N}=3$ missing) } \\
\hline Fall & $74(89.2 \%)$ \\
\hline Direct trauma & $7(8.4 \%)$ \\
\hline Pulled elbow mechanism & $2(2.4 \%)$ \\
\hline \multicolumn{2}{|l|}{ Polytrauma ( $\mathrm{N}=2$ missing) } \\
\hline No & $80(95.2 \%)$ \\
\hline Yes & $4(4.8 \%)$ \\
\hline \multicolumn{2}{|l|}{ Associated injuries } \\
\hline Hand & $1(1.2 \%)$ \\
\hline Wrist & $2(2.3 \%)$ \\
\hline \multicolumn{2}{|l|}{ Dislocation side } \\
\hline Left & $59(68.6 \%)$ \\
\hline Right & $27(31.4 \%)$ \\
\hline \multicolumn{2}{|l|}{ Type of dislocation ( $\mathrm{N}=18$ missing) } \\
\hline Anterior & $1(1.5 \%)$ \\
\hline Posterior & $30(44.1 \%)$ \\
\hline Posteromedial & $6(8.8 \%)$ \\
\hline Posterolateral & $31(45.6 \%)$ \\
\hline \multicolumn{2}{|l|}{ Fracture $(\mathrm{N}=1$ missing $)$} \\
\hline No & $43(50.6 \%)$ \\
\hline Yes & $42(49.4 \%)$ \\
\hline \multicolumn{2}{|l|}{ Fracturetype } \\
\hline Coronoid fracture & 18 \\
\hline Radial head fracture & 24 \\
\hline Humerus & 5 \\
\hline Olecranon & 3 \\
\hline Coronoid + radial head fracture & 4 \\
\hline Sensibility deficit & $2(2.3 \%)$ \\
\hline Vascular injury & $1(1.2 \%)$ \\
\hline
\end{tabular}

In $54 \%(n=25)$ of the cases the dislocation was on the nondominant side; this tendency compared with the dominant side was insignificant $(\mathrm{p}=0.63)$. Complex dislocations were diagnosed in $49 \%$ of the patients $(n=42)$. The accompanying fractures were categorised as follows: coronoid $n=18$, radial head $n=24$, humerus $n=5$, olecranon $n=3$. Four patients sustained a coronoid fracture as well as a radial head fracture, the so-called terrible triad. A total of six radial head fractures were operated on, of which three received open reduction and internal fixation (ORIF), one received radial head resection, one a partial radial head resection, and one a prosthesis (because of re-dislocation after one week in plaster). One coronoid fracture was treated with ORIF 
(AO screw). Thirty-five small avulsions were not operated on. One loose body within the joint due to a coronoid fracture necessitated removal.

A total of three dynamic fixators were mounted, one for medial instability, one due to re-dislocation in plaster (both of which were simple elbow dislocations) and one as functional treatment (complex elbow dislocation). We recorded 4 cases of re-dislocation. One re-dislocation occurred after sufficient trauma three months after the primary dislocation. Routine X-Ray examination after one week in plaster detected another two. One of these had an associated radial head fracture and received radial head prosthesis. In the other one a functional external fixator was applied (as stated above). The fourth patient with a redislocation was diagnosed after four weeks and the redislocation was reduced surgically.

Dislocation was primarily reduced at the emergency department in $72(84 \%)$ and in the theatre in $14(16 \%)$ cases. In 8 of the 72 cases primarily reduced at the emergency department, the reduction did not succeed and these elbows were then reduced secondarily in the operating theatre. In 18 patients, stability testing was recorded in the medical history.

In two of the 18 patients, the collateral ligaments were sutured. Seventy-four patients were either exclusively treated with a plaster cast or started with a plaster cast followed by functional treatment in a hinged brace $(\mathrm{n}=23)$, sling $(\mathrm{n}=1)$ or pressure bandage $(n=4)$.

Three patients had only a pressure bandage, three patients a sling and one patient a brace and sling. Thirty-two percent of the patients had a plaster immobilisation longer than three weeks and $49 \%$ of the elbows were immobilised for three weeks or longer.

Two cases presented with a sensory deficit and one had a vascular injury. One patient required an ulnar nerve release.

The median period of treatment was 6.0 weeks $(\mathrm{IQR}=7)$. The mean period in plaster was 2.6 weeks $(\mathrm{SD}=1.7)$.

Six weeks after reduction of the elbow dislocations, the mean extension loss was $18.3^{0}(\mathrm{SD}=19.0)$ and the following range of motions were found: The mean flexion was $131.0^{0}$ $(\mathrm{SD}=20.8)$, pronation $79.9^{\circ}(\mathrm{SD}=21.8)$, and supination $78.2^{0}$ $(\mathrm{SD}=22.9)$. No statistically significant differences were found for range of motions (ROM) between patients with versus without fractures. The duration of plaster immobilisation had no correlation with the range of motions at the end of the treatment and at long-term follow-up.

The time between the dislocation and the follow-up examination was $3.3(\mathrm{SD}=2.6)$ years. The results of this examination are presented in Table 2 . No statistically significant differences were found between patients with versus without fracture (independent t-test). The Pearson correlation between the duration of plaster immobilisation and the Oxford function score were significant for the whole group $(\mathrm{r}=-0.40, \mathrm{p}=0.006, \mathrm{n}=46)$ and for the patients without fractures $(\mathrm{r}=-0.58, \mathrm{p}=0.004, \mathrm{n}=22)$. The Pearson correlation for the whole group $(n=46)$ between the duration of plaster immobilisation and the Oxford pain score was -0.37 $(\mathrm{p}=0.012)$ and with the Oxford social-psychological score was $-0.37(\mathrm{p}=0.011)$.
Table 2. An Overview of the Results after a Mean Follow-Up of 3.3 Years $(\mathrm{SD}=2.6)$

\begin{tabular}{|l|l|}
\hline \multicolumn{1}{|c|}{ Range of Motion } & \multicolumn{1}{|c|}{ Mean (SD) } \\
\hline \hline Extension loss ( $\mathrm{n}=42)$ & $5.5^{\circ}(11.0)$ \\
\hline Flexion ( $\mathrm{n}=42)$ & $141.0^{\circ}(7.5)$ \\
\hline Pronation ( $\mathrm{n}=36)$ & $89.7^{\circ}(1.7)$ \\
\hline Supination (n=36) & $88.3^{\circ}(8.5)$ \\
\hline Pain Visual Analogue Scale - VAS $(\mathrm{n}=50)$ & $1.98(7.0)$ \\
\hline MEPI total score (max 100 points) $\mathrm{n}=40$ & $91.9(11.8)$ \\
\hline MEPI pain score (max 45) & $37.5(11.8)$ \\
\hline MEPI ulnohumeral motion (max 20) & $19.8(1.1)$ \\
\hline MEPI stability (max 10) & $9.6(1.3)$ \\
\hline MEPI function (max 25) & $25(0)$ \\
\hline MEPI categories ( $\mathrm{n}=40):$ & \\
Excellent (90-100 points) & $26(65 \%)$ \\
\hline Good (75-89 points) & $9(22.5 \%)$ \\
\hline Fair (60-74 points) & $4(10 \%)$ \\
\hline Poor (<60 points) & $1(2.5 \%)$ \\
\hline Quick DASH questionnaire: & \\
\hline Disability score (n=59) & $9.7(13.0)$ \\
\hline Sports/music score ( $\mathrm{n}=44)$ & $11.5(19.2)$ \\
\hline Work activities ( $\mathrm{n}=49)$ & $6.1(12.4)$ \\
\hline Oxford elbow score $(\mathrm{n}=57):$ & $75.7(27.1)$ \\
\hline Elbow function & $75.2(28.3)$ \\
\hline Pain & $73.9(26.6)$ \\
\hline Social-psychological & \\
\hline
\end{tabular}

\section{DISCUSSION}

Conn et al found 414 injuries of the elbow in their fracture service, including 58 elbow dislocations in children and adults [9]. Elbow injuries accounted for $6.8 \%$ of all treated fractures. Seventy-six percent of the patients with elbow dislocations were older than 20 years. In $51 \%$ of these adults, the dislocations were simple, a lower percentage than the $74 \%$ found in Josefsson's study [1]. In another study by Josefsson, $46 \%$ of the patients with simple dislocation were 16 years or older [10]. In our study $52 \%$ of the dislocations were of the simple type however, in our study only patients of 18 years and older were selected.

Elbow dislocations are defined by the direction of their displacement. Nearly all the dislocations are of the posterior or posterolateral types. In Conn's study, $96 \%$ of the dislocations were posterior or lateral [9] and Joseffson reported no anterior dislocations in his study of 52 patients [10]. In our study $89.7 \%$ of the dislocations were of the posterior or posterolateral type and $1.5 \%$ were of the anterior type.

In a case-control study only $6 \%$ of a studied American population $(\mathrm{n}=5033)$ was left handed and left-handedness was a risk factor for fractures [11]. In another study fractures proximal to the wrist occurred more frequently on the left side, but were unrelated to dominance [12]. In $58 \%$ of the patients of Joseffson, the simple elbow dislocations were on the nondominant left side [10]. In our study $69 \%$ of the dislocations were found on the left side. In our patients records dominance 
was in $47 \%$ not recorded, but in the recorded cases $54 \%$ of the dislocations were on the non-dominant side. This occurrence was non-significant. The exact cause of the preponderance of elbow dislocations on the left side is unknown, but it is probably not related to dominance.

Following reposition and treatment in plaster of simple dislocations, recurrent dislocations and chronic instability are rare [2, 13]. In Joseffson's study one obviously unstable joint was described in his study of 52 patients after a mean follow-up of 24 years [10]. We observed four redislocations (3\%), however, one redislocation was caused by an adequate trauma and was not considered a result of persistent instability. Of the three remaining patients, two had a complex elbow dislocation with a radial head fracture, which was primarily left untreated and one polytrauma patient had probably an unstable elbow after reduction.

In theory, after repositioning of a simple dislocated elbow, the joint retains an inherent stability caused by the contour of the intact joint surfaces. This stability may allow the patient to exercise the joint shortly after the repositioning. In these cases, functional treatment may enhance recovery of a full range of motion without risking increased joint instability. Nearly half of our patients were immobilised in plaster three weeks or longer. Shorter periods of plaster immobilisation after reduction of elbow dislocations has been associated with better range of motions. Protzmann [14] describes less extension loss and shorter mean disability in weeks for the shorter immobilisation group without making statistical inferences. Mehlhoff [15] describes less extension loss for the shorter immobilisation groups, with a significant correlation between extension loss and duration of follow-up. He also reported less flexion loss and less prevalence and severity of pain for the shorter immobilisation groups but did not analyze this data statistically. The number of patients with symptoms of instability of the elbow joints was lower in the shorter immobilisation group without reaching statistical significance at the $5 \%$ level. Schippinger [16] described better Morrey scores, which are composed of the items pain, movement, strength, instability and function (activities of daily living), and better separate pain scores in the shorter immobilisation groups, though without statistical significance. In our study, there was no correlation for the entire patient group between range of motion and duration of immobilisation, which lasted three weeks or longer for $49 \%$ of the patients. However, the weak correlation between the duration of immobilisation and the three Oxford elbow scores after a mean of 3.3 years was negative and significant; so, the shorter the immobilisation, the better the Oxford elbow scores were. This is probably due to methodological bias: The simpler cases got shorter immobilisation. Furthermore, even in the patient group without fractures, these correlations were moderate. In a retrospective analysis this is probably due to methodological bias: The simpler cases got shorter immobilisation.
Our patient sample consisted mainly (92\%) of elbow dislocations without or with small avulsions. Stability was not routinely tested and was only in $21 \%$ recorded in the hospital charts. Serious injury of the collateral ligaments was not described. So our prudent conclusions are probably only relevant to the simple stable elbow dislocations. Our results seem to suggest that elbow dislocation with and without small fractures is a mild condition that can be treated with good results. Functional treatment and early active movement within the limits of pain can probably improve the final Oxford elbow score.

\section{REFERENCES}

[1] Josefsson PO, Nilsson BE. Incidence of elbow dislocation. Acta Orthop Scand 1986; 57(6): 537-8.

[2] Hildebrand KA, Patterson SD, King GJ. Acute elbow dislocations: Simple and complex. Orthop Clin North Am 1999; 30(1): 63-79.

[3] Morrey BF, An KN, Chao EYS. Functional evaluation of the elbow. In: Morrey BF, Ed. The Elbow and Its Disorders. $2^{\text {nd }}$ ed. Philadelphia: WB Saunders; 1993. pp. 86-9.

[4] Beaton DE, Katz JN, Fossel AH, Wright JG, Tarasuk V, Bombardier C. Measuring the whole or the parts? Validity, reliability, and responsiveness of the disabilities of the arm, shoulder and hand outcome measure in different regions of the upper extremity. J Hand Ther 2001; 14(2): 128-46.

[5] Hudak PL, Amadio PC, Bombardier C. Development of an upper extremity outcome measure: The DASH (disabilities of the arm, shoulder and hand) [corrected]. The Upper Extremity Collaborative Group (UECG). Am J Ind Med 1996; 29(6): 602-8.

[6] Dawson J, Doll H, Boller I, et al. The development and validation of a patient-reported questionnaire to assess outcomes of elbow surgery. J Bone Joint Surg Br 2008; 90(4): 466-73.

[7] Dawson J, Doll H, Boller I, et al. Comparative responsiveness and minimal change for the Oxford Elbow Score following surgery. Qual Life Res 2008; 17(10): 1257-67.

[8] Floor S, Overbeke AJ. [Questionnaires on the quality of life in other than the Dutch language used in the Nederlands Tijdschrift voor Geneeskunde (Dutch Journal of Medicine): The translation procedure and arguments for the choice of the questionnaire]. Ned Tijdschr Geneeskd 2006; 150(31): 1724-7.

[9] Conn J Jr, Wade PA. Injuries of the elbow: A ten year review. J Trauma 1961; 1: 248-68.

[10] Josefsson PO, Johnell O, Gentz CF. Long-term sequelae of simple dislocation of the elbow. J Bone Joint Surg Am 1984; 66(6): 927-30.

[11] Luetters CM, Kelsey JL, Keegan TH, Quesenberry CP, Sidney S. Lefthandedness as a risk factor for fractures. Osteoporos Int 2003; 14(11): 918-22.

[12] Meals RA. The laterality of fractures and dislocations with respect to handedness. Clin Orthop Relat Res 1979; (143): 158-61.

[13] De Haan J, Schep NW, Tuinebreijer WE, Patka P, den Hartog D. Simple elbow dislocations: A systematic review of the literature. Arch Orthop Trauma Surg 2009; 130: 241-9.

[14] Protzman RR. Dislocation of the elbow joint. J Bone Joint Surg Am 1978; 60(4): 539-41.

[15] Mehlhoff TL, Noble PC, Bennett JB, Tullos HS. Simple dislocation of the elbow in the adult. Results after closed treatment. J Bone Joint Surg Am 1988; 70(2): 244-9.

[16] Schippinger G, Seibert FJ, Steinbock J, Kucharczyk M. Management of simple elbow dislocations. Does the period of immobilization affect the eventual results? Langenbecks Arch Surg 1999; 384(3): 294-7. 